\title{
Factores asociados al riesgo en la deserción estudiantil en un Instituto de Educación Superior Tecnológico Público
}

\author{
Factors associated with risk of student dropout in a Public Technological \\ Institute of Higher Education
}

Juan Mori Quiroz (ORCID 0000-0002-0452-5862)1

${ }^{1}$ Universidad Cesar Vallejo

\begin{abstract}
Resumen
Esta investigación tiene como objetivo determinar la influencia de los factores asociados al riesgo en la deserción estudiantil de un instituto superior tecnológico público. El interés en este tema es fruto de una problemática real como la deserción estudiantil, que a su vez constituye un tema de mucho cuidado para los estudiantes de institutos tecnológicos. La investigación es de naturaleza cuantitativa, su nivel es correlacional causal, y es de diseño no experimental, con una población de 131 estudiantes que desertaron en el año 2018. La muestra fue de 80 estudiantes y se han considerado criterios de inclusión y exclusión. Se empleó la técnica llamada encuesta y el instrumento utilizado fue un cuestionario de 31 preguntas, 16 para la variable factores de riesgo y 15 para la variable deserción. El resultado relevante es que la dimensión socioeconómica es la de mayor significación $(p=0,005<0,05)$ para la deserción estudiantil. La conclusión es que las otras dimensiones personales, académicas e institucionales no inciden en la deserción, sino que son útiles para definir actividades preventivas para que, en un futuro, no influyan en esta.
\end{abstract}

Palabras clave: Factores de riesgo, deserción estudiantil, influencia.

Fecha de envío: 15/07/2021

Fecha de aceptación: 03/08/2021 


\begin{abstract}
This research aims to determine the influence of factors associated with risk in student dropout from a public higher technological institute. Interest in this topic is the result of a real problem such as student desertion, which in turn constitutes a very careful topic for students of technological institutes. The research is quantitative in nature, its level is causal correlational, and it is of a non-experimental design, with a population of 131 students who dropped out in 2018. The sample was 80 students and inclusion and exclusion criteria have been considered. The technique called survey was used and the instrument used was a questionnaire with 31 questions, 16 for the risk factors variable and 15 for the attrition variable. The relevant result is that the socioeconomic dimension is the most significant $(p=0.005<0.05)$ for student dropout. The conclusion is that the other personal, academic and institutional dimensions do not affect dropout, but are useful to define preventive activities so that, in the future, they do not influence it.
\end{abstract}

\title{
Keywords: Risk factors, student desertion, influence.
}

\section{Introducción}

En los últimos años, la deserción estudiantil se ha convertido en tema de mucha preocupación para las autoridades de los Institutos Tecnológicos y, a su vez, para otros especialistas. La deserción no solo debe ser materia de estudio del sector educación, sino que debe ser enfocada desde una perspectiva multifactorial, dicho sea, con consideración de los puntos de vista económico, social y pedagógico.

A nivel internacional (López, 2018), se produjo un cambio de 13 millones de alumnos matriculados en el año 1960 a 198 millones en el año 2013. La tasa de deserción a nivel mundial bordea el $38 \%$. En Latinoamérica y el Caribe, el registro de estudiantes cambia de un país a otro: Argentina con $80 \%$; Chile, $79 \%$; Cuba, $48 \%$; Colombia, $48 \%$; México, $30 \%$; Jamaica, $29 \%$. En el año 2006, se encargó al Cen- tro Interuniversitario de Desarrollo (CINDA, 2006) de Chile y al IESAL- UNESCO (Trevellini, 2018) realizar un estudio sobre la deserción, el cual tuvo como resultado que el porcentaje de estudiantes que no culminaban sus estudios estaba alrededor del $57 \%$. Esta tendencia todavía se mantiene en la actualidad.

Actualmente, a nivel de Institutos de Educación Superior en el Perú se tuvieron más de 400 mil estudiantes cursando estudios en educación superior tecnológica ubicados en las regiones de Lima, Arequipa, Cusco, La Libertad, Junín y Lambayeque. Lima concentra una mayor cantidad con 198916 estudiantes, pero alrededor de $36 \%$ (71446 estudiantes) no se graduaron. Además, más de 90000 mil estudiantes abandonan sus estudios superiores cada año a nivel de la región Lima. De esta cantidad, el $70 \%$ son de instituciones con gestión particular y el $30 \%$ rema- 
nente son de establecimientos del Estado (SINEACE, 2018). Algo similar sucede en las demás regiones. El INEI, en el Censo Nacional 2017, publicó que se pierden aproximadamente 1,150 millones de soles anuales (Instituto nacional de estadística e informática, 2017), lo cual está siendo desaprovechado por los estudiantes, puesto que no culminan el año, repiten el ciclo o dejan el semestre de estudio.

En el contexto local en el Instituto de Educación Superior Tecnológico Público, se reportó que en el año 2017 se retiraron 117 estudiantes de 2701 estudiantes matriculados. Asimismo, en el año 2018 se matricularon 2823 y se retiraron 131 estudiantes; se produjo un pequeño incremento por la creación de la carrera Desarrollo de Sistema de Información. Los estudiantes que desertaron podrían pasar a formar parte de esa masa de 1500000 jóvenes en el Perú que no estudia ni trabaja, y que por lo cual constituye una carga para la familia y un problema latente para la sociedad. Existe ciertamente desmotivación, desinterés y falta de orientación hecha por una carrera o institución que se adecúe al perfil del alumno que satisfaga sus expectativa. También podrían influir la incomodidad de sus aulas, el desfase tecnológico de sus talleres o de sus planes curriculares que no han sido adaptados al desarrollo de nuevas tecnologías.

La investigación se respalda en la revisión de trabajos previos a nivel nacional e internacional. A nivel nacional, Corzo (2018) encontró que existe, en su investigación correlacional sobre la dirección administrativa y el abandono de los estudiantes en un instituto de educación superior tecnológico, una conexión entre la di- rección administrativa y la deserción. Asimismo, Ruiz (2018), a partir de su estudio sobre el abandono de alumnos del primer ciclo en el Instituto de Administración Señor de Sipán, concluyó que los aspectos personales y tiempo dedicados al estudio inciden en el abandono de clase. En su estudio sobre retención de estudiantes, Sagenmüller (2019) encuentra razones por las cuales las personas abandonan la educación superior y concluye que, según cual sea la institución educativa, existen factores comunes que desencadenan el abandono en la educación superior. Estos pueden ser problemas personales como también una conjunción de diversos problemas, que de no ser detectados oportunamente por el personal administrativo de la institución, podrían generar una disminución en la retención de los estudiantes.

A nivel internacional, Merlos (2017) concluye, a partir de una investigación sobre la deserción escolar en estudiantes no tradicionales en centros de capacitación de México, que existe una mayor deserción cuando los estudiantes no tienen tiempo para ir a los centros de capacitación debido a que colisionan las horas de trabajo con las horas de estudio. Asimismo, Casanova, Cervero, Nuñez, Almeida y Bernardo (2018), en un estudio sobre factores que determinan la persistencia y deserción de los estudiantes universitarios en España, concluyeron que el rendimiento académico es concluyente en la toma de decisión de continuar o abandonar los estudios. En este caso, la decisión de permanencia del $95.8 \%$ es mayor a la de deserción con un $65.6 \%$. Albán y Mauricio (2018), en una investigación sobre factores que influyen en la deserción universitaria 
en Ecuador, concluyen que las probabilidades de deserción son altas con un $57 \%$. Los factores más notorios son el número de hijos de los estudiantes y las perspectivas de los estudiantes sobre su integración en el mercado.

Los conceptos teóricos nos permitieron sustentar la investigación. Uno de ellos es la variable factor de riesgo según (Bonilla, 2006, citado en Jiu, 2016) "Es un conjunto de situaciones, circunstancias o características: personales, sociales, económicos, culturales y otros, el cual constituye un patrón de vulnerabilidad para el adolescente" (p.26). Por otro lado, (Pérez y Gardey, 2017, pp.1-2) definió un factor como un elemento que actúa en conjunto con otros. La idea de riesgo, por su parte, alude a la cercanía o la inminencia de un daño. Existen diferentes factores de riesgo tales como: Factores de riesgo conductuales están ligados a ciertas acciones que la persona decide llevar a cabo, por lo cual deben ser reducidos o eliminados ya que afecta su conducta o estilo de vida. Factores de riesgo demográficos caracterizan a los individuos que forman una población como el género, su actividad laboral, el sueldo, la edad, estado civil y su credo religioso.

En lo que respecta a la variable deserción, Rodríguez y Hernández (Torres et al, 2016) aseguraron que los factores varían en función del contenido y características académicas, individuales, socioeconómicas y organismos educativos. Según SCALE (MINEDU, 2014, pp. 1-2), la deserción estudiantil se entiende "como el abandono del sistema educativo por parte de los alumnos que habiendo estado matriculados ya no lo hacen al año siguiente, no completando estos su educación primaria o secundaria por uno o más períodos educativos". Asimismo, Rojas (2008) lo considera como el abandono voluntario o forzado de la carrera en la que el estudiante se matriculó. El fenómeno en cuestión se produce cuando se retira de la institución sin completar un programa académico (2008, pp. 86-87).

Respecto a las teorías sobre la variable estudiada, Viale (2014) expone un conjunto de teorías y su incidencia en el abandono de los estudios: las teorías psicológicas, sociológicas, económicas e interaccionistas. Las teorías psicológicas relacionan las teorías de la personalidad con el abandono sin considerar el comportamiento del estudiante. Por otro lado, en el caso de las teorías sociológicas, estas relacionan aspectos externos al individuo que inciden en la deserción y consideran fenómenos sociales sin tener en cuenta la influencia de la institución. Las teorías económicas responden a la relación costo-beneficio de los estudiantes, es decir, la causa económica es razón para tomar la decisión de desertar. Y, respecto a las teorías interaccionistas, estas utilizan teorías que comparan características de los estudiantes con el entorno que ofrece la institución (Viale, 2014, pp. 5-6).

Asimismo, Fonseca y García (2016) señalan en su enfoque sobre las teorías organizacionales que las características de la institución educativa, como el servicio que ofrece a los estudiantes, podrían también influir en la deserción de los estudiantes.

Debemos considerar que la investigación se realizó en el periodo del 2017 al 2018 en el que los factores de la deserción estudiantil eran diferentes a los que 
existen hoy en el contexto de pandemia del COVID-19, puesto que en este último la situación económica de los hogares y los problemas de conectividad que viven los estudiantes son los factores predominantes. En el periodo que hemos considerado hay ciertamente otros factores, pero no dejan de ser relevantes el factor económico, los problemas de la sociedad y la cultura dentro del área de cobertura de la institución, y estos seguirán a pesar de la situación de salud que atravesamos, así como los posibles cambios estructurales o administrativos que puedan producirse en estas instituciones de educación superior. Conocer la problemática de la deserción y los niveles de deserción escolar que se obtengan deberá permitir a las autoridades educativas plantear estrategias que busquen mejorar la retención estudiantil, así como mejorar el trabajo administrativo en las instituciones e incentivar un mejor trabajo en el aula al perfeccionar la comunicación docente-alumno. La deserción estudiantil llega a tener un costo social elevado, por lo que identificar sus causas o factores debe permitir sugerir medidas preventivas desde la perspectiva de la institución educativa, con la finalidad de minimizarlo y generar un ambiente más acogedor donde los estudiantes encuentren mejores condiciones a fin de desarrollar un proceso de enseñanza aprendizaje de mejor calidad y con el compromiso de una mejora continua.

Sobre la base de lo anterior se plantea este problema general: ¿De qué modo influyen los factores asociados al riesgo en la deserción estudiantil del instituto superior tecnológico público?

La hipótesis general (H1) es que los fac- tores asociados al riesgo influyen en la deserción estudiantil del instituto superior tecnológico público. En este trabajo se pretende alcanzar el objetivo es determinar la influencia de los factores asociados al riesgo en la deserción estudiantil del instituto superior tecnológico público.

\section{Materiales y método}

En la investigación se ha considerado el método hipotético-deductivo, el cual según Econlink (2017) consiste en definir pronósticos que deben ser ciertos si el supuesto es verdadero; busca relacionar los pronósticos con una exploración empírica y sobre la base de ello acepta o rechaza una teoría. A su vez, el enfoque es cuantitativo; según McLeod (2019), este consiste en levantar datos como números para luego ordenarlos en clases o en unidades de medida. Se empleó también el tipo de investigación básica. Según Valderrama (2013, p. 124), esta está destinada a aportar un cuerpo organizado de conocimientos científicos y no produce necesariamente resultados de utilidad práctica inmediata. Del nivel descriptivo, Bernal (2006) sostuvo que los estudios descriptivos miden de forma independiente las variables, aun cuando no se formule hipótesis alguna. Si en estas hay profundidad se abordará el objeto, sujeto o fenómeno a estudiar (2006, p. 98).

Se utilizó un diseño no experimental, correlacional causal y transversal. Para Way, L. (2018), esta es una investigación en la que se puede describir un evento o acontecimiento tal como se encuentra, sin que el investigador pueda intervenir; 
Tabla 1: Frecuencias de categorización de la variable factores de riesgos y deserción en estudiantes del Instituto Superior Tecnológico Público

\begin{tabular}{lll}
\hline Factores de riesgos & Frecuencia & Porcentaje válido \\
\hline Riesgo bajo & 4 & 5.3 \\
Riesgo medio & 69 & 92.0 \\
Riesgo alto & 2 & 2.7 \\
Total & 75 & 100.0 \\
Deserción & Frecuencia & Porcentaje valido \\
Deserción baja & 3 & 4 \\
Deserción media & 52 & 69.3 \\
Deserción alta & 20 & 26.7 \\
Total & 75 & 100.0 \\
\hline
\end{tabular}

es decir, no puede manipular la variable (Lani, 2008, p. 2). Sánchez y Reyes (2002, p. 79) sostuvieron que el estudio correlacional causal "[e]stá orientado a la determinación del grado de relación existente entre dos o más variables de interés de una misma muestra". Es de corte transversal debido a que se recogen datos en un solo tiempo. La población, según Rafeedali (2018, p. 1), "está reproducida por un conjunto repleto de personas, instituciones, objetos, etc., con particularidades afines que son de interés del investigador". En este caso la población corresponde a 131 estudiantes que desertaron de la institución durante el año 2018 (I y II ciclo). La muestra corresponde a 80 alumnos, los cuales fueron contactados y accedieron a participar. Se determinó el tamaño de la muestra mediante el muestreo no probabilístico. El instrumento utilizado para medir ambas variables fue un cuestionario de 31 preguntas y de autoría propia, dividido en 16 preguntas para la variable factores de riesgo y distribuidas en cuatro dimensiones: factores personales, académicos, socioeconómicos e institucionales. 15 preguntas fueron para la variable deserción definidas en tres dimensiones: relación con la institución, retiro temporario o definitivo de la institución.

La técnica utilizada fue la encuesta y el método de medición la escala de Likert que evalúa actitudes y opiniones de los estudiantes. El grado en que un instrumento realmente mide la variable -es decir, la validez- se determinó mediante el juicio de expertos para ambas variables. La fiabilidad del instrumento utilizado para la prueba fue estimada mediante el coeficiente alfa de Cronbach: para la variable factores de riesgo fue 0,740 y para la variable deserción 0,732 . La prueba piloto de los dos instrumentos fue mayor a 0,71. Podemos decir entonces que ambos instrumentos fueron confiables.

\section{Resultados}

\subsection{Resultados descriptivos}

Como puede verse en Tabla 1 y Figura 1 , se observó una frecuencia dominante absoluta en la categoría de riesgo medio 




Figura 1: Niveles de categorización de la variable factores de riesgos y deserción en estudiantes del Instituto de Educación Superior Tecnológico Público

Tabla 2: Frecuencias de categorización de las dimensiones de la variable factores de riesgos y deserción en estudiantes del Instituto de Educación Superior Tecnológico Público.

\begin{tabular}{llllllll}
\hline Dimensiones de los factores & \multicolumn{2}{l}{ Riesgo bajo } & \multicolumn{2}{c}{ Riesgo medio } & \multicolumn{2}{c}{ Riesgo alto } \\
de riesgo y de la deserción & $\mathbf{n}$ & $\%$ & $\mathbf{N}$ & $\%$ & N & $\%$ \\
\hline Factores Personales & 13 & 17.3 & 57 & 76.0 & 5 & 6.7 \\
Factores Académicos & 20 & 26.7 & 50 & 66.7 & 5 & 6.7 \\
Factores Institucionales & 4 & 5.3 & 59 & 78.7 & 12 & 16.0 \\
Factores socioeconómicos & 17 & 22.7 & 46 & 61.3 & 12 & 16.0 \\
Relación con la institución & 5 & 6.7 & 42 & 56.0 & 28 & 37.3 \\
Retiro temporal o definitivo de la & 9 & 12.0 & 52 & 69.3 & 14 & 18.7 \\
Institución & & & & & & \\
Descontento con la institución & 5 & 6.7 & 32 & 42.7 & 38 & 50.7 \\
\hline
\end{tabular}

con un $92 \%$. Tanto las categorías riesgo bajo como riesgo alto fueron muy bajos, teniendo $5.3 \%$ y $2.7 \%$ respectivamente. Se notó que la mayor frecuencia estuvo en la categoría deserción media, con un $69.3 \%$, así como con una tendencia definida hacia una alta deserción $(26.7 \%)$. Por otra parte, la deserción baja solamente tuvo un $4 \%$.
En la Tabla 2 y Figura 2 se aprecia en todas las dimensiones que predominó el riesgo medio. En la dimensión de factores socioeconómicos tuvo el menor porcentaje $61.3 \%$ y la dimensión factores institucionales obtuvo el mayor porcentaje $78.7 \%$. En ambas se alcanzó el mayor porcentaje en riesgo alto, ambos un $16 \%$. En la variable deserción, respecto a las dos primeras 


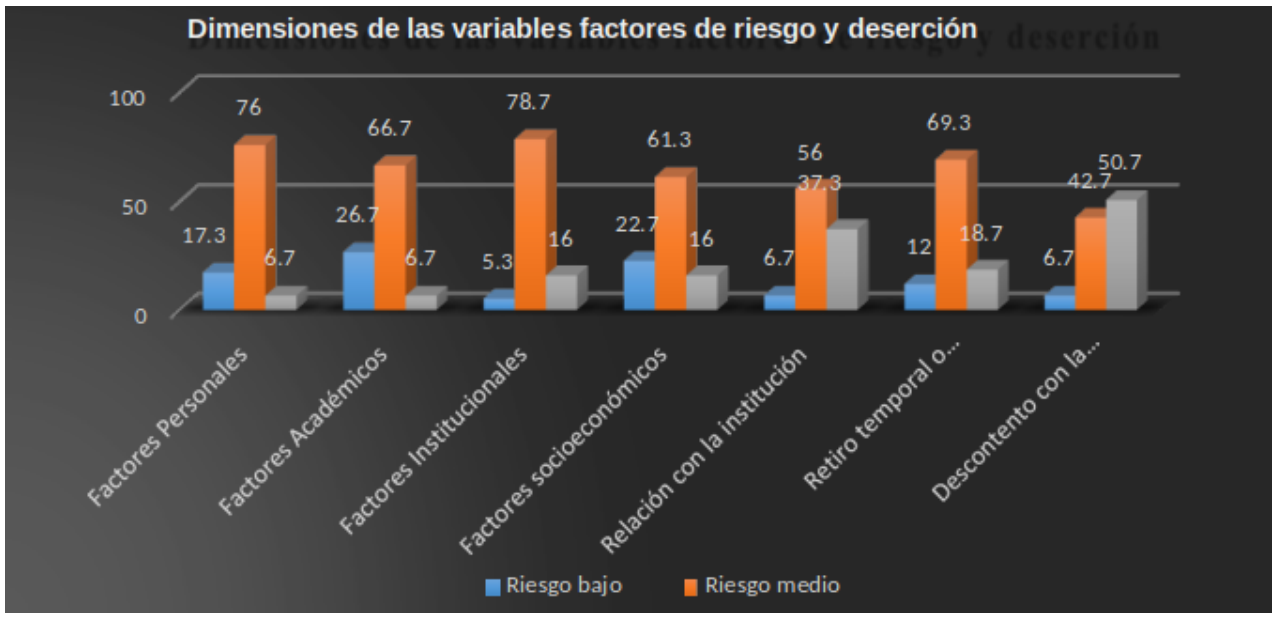

Figura 2: Niveles de categorización de las dimensiones de las variables factores de riesgo y deserción en estudiantes del Instituto de Educación Superior Tecnológico Público

dimensiones se observó una deserción media con un $56 \%$ y $69.3 \%$. En la dimensión descontento con la institución se observó un $50.7 \%$ en la categoría deserción alta, siendo esta la mayor de todas.

\subsection{Resultados inferenciales (prueba de la hipótesis)}

En la Tabla 3 se observó que el valor de la significancia del Chi cuadrado de Pearson fue 0.340 , cantidad que es mayor al valor permisible 0.05 . Teniendo en consideración que la Bondad de ajuste es una prueba requisito, el valor superior es un buen indicador gracias al cual se tiene evidencia para señalar que el modelamiento con la regresión logística se adecúa para este análisis de regresión.

En la Tabla 4, este resultado revela que, si bien el modelamiento de regresión logística ordinal podría explicar la asociación entre estas dos variables, no obstante, el porcentaje de potencia es bajo pe- ro considerable, oscilando entre $16.8 \%$ a $021.8 \%$, como lo revelan los coeficientes de Cox y Snell y de Nagelkerke respectivamente; se interpreta como el intervalo de potencia de predicción de este modelo. Así, se plantea recién las hipótesis de contraste:

H1: Los factores asociados al riesgo influyen en la deserción estudiantil del Instituto Superior Tecnológico Público.

H0: Los factores asociados al riesgo no influyen en la deserción estudiantil del Instituto Superior Tecnológico Público.

En la Tabla 5 se observó que solamente en la categoría deserción baja hubo significación $(p=0,005<0,05)$, en contraste con el nivel deserción media que no lo fue. Respecto a la variable independiente (Factores de riesgo), se vio que ninguno de los tres niveles fue significativo, por lo cual no hubo evidencia para rechazar la hipótesis nula afirmando que los factores asociados al riesgo no influyen en la deserción estudiantil del Instituto. Este resultado se 
Tabla 3: Bondad de ajuste de la prueba de regresión entre los factores asociados sobre la deserción estudiantil Instituto Superior Tecnológico Público

\begin{tabular}{llll}
\hline & Chi-cuadrado & Gl & Sig. \\
\hline Pearson & 2,159 & 2 &, 340 \\
Desvianza & 1,832 & 2 &, 400 \\
\hline
\end{tabular}

Función de enlace: Logit.

Tabla 4: Pseudo R cuadrado de la prueba de regresión entre los factores asociados sobre la deserción estudiantil del Instituto Superior Tecnológico Público

\begin{tabular}{ll}
\hline Cox y Snell &, 168 \\
Nagelkerke &, 218 \\
McFadden &, 115 \\
\hline
\end{tabular}

Función de enlace: Logit.

vio refrendado por el coeficiente de Wald, el cual estadísticamente podría ser equivalente a un cero, ya que su p-valor es mayor que 0.05 . Por lo tanto, la variable independiente no influye a la hora de determinar la variable dependiente del modelo de regresión. Con este resultado no concluyente tenemos que la interacción de los niveles bajo y medio de los factores podría influenciar en la deserción. Ampliaríamos el estudio con mayor número de unidades de análisis.

\subsection{Hipótesis específica}

En la Tabla 6 vemos que el valor de la significancia del Chi cuadrado de Pearson fue 0.175 , cantidad mayor que la permitida (0.05), y teniendo en cuenta que la Bondad de ajuste es una prueba requisito, el valor superior es un buen indicador por el cual hay evidencia para señalar que posiblemente el modelamiento con la regresión logística se adecúe para este análisis de regresión.

Por su parte, en la Tabla 7 puede ver- se que este resultado revela que, si bien el modelamiento de Regresión logística ordinal podría explicar la asociación entre los factores y la variable dependiente, no obstante, el porcentaje de potencia es bajo pero considerable, ya que oscila entre $13.9 \%$ a $18.1 \%$, como lo revelan los coeficientes de Cox y Snell y de Nagelkerke respectivamente. Esto se interpreta como el intervalo de potencia de predicción de este modelo.

\subsection{Regresión logística ordinal de las dimensiones de la va- riable factores de riesgo so- bre la deserción}

En la Tabla 8 se observó que ninguno de los niveles de las variables independientes fue significativo, ya que los valores fueron superiores al nivel propuesto. Los factores de riesgo personales, institucionales, académicos y socioeconómicos no influyen en la deserción de estudiantes. Y, a pesar de que tampoco el estudio no es conclu- 
Tabla 5: Estimación de parámetros mediante la prueba de regresión logística ordinal de la variable Factores de riesgo sobre la deserción estudiantil del Instituto Superior Tecnológico Público

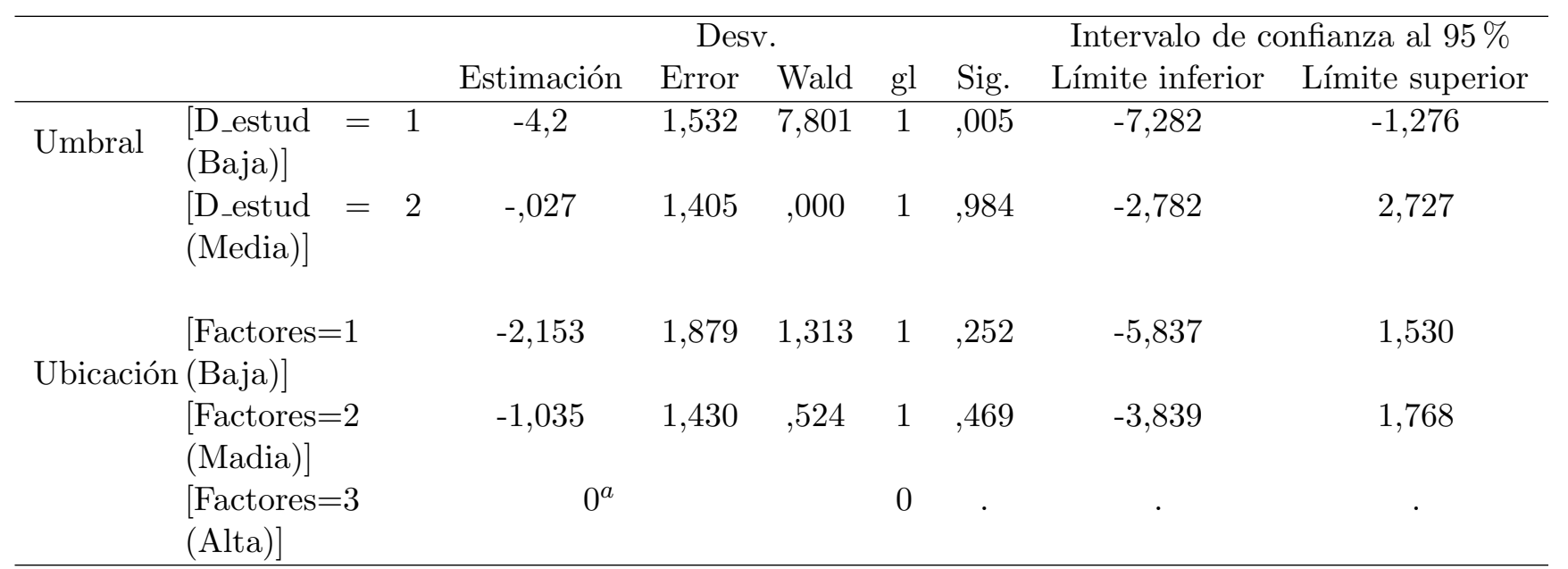

Función de enlace: Logit.

${ }^{a}$ Este parámetro está establecido en cero porque es redundante.

Tabla 6: Bondad de ajuste de la prueba de regresión entre las dimensiones de los factores asociados al riesgo sobre la deserción estudiantil del Instituto Superior Tecnológico Público

\begin{tabular}{llll}
\hline & Chi-cuadrado & gl & Sig. \\
\hline Pearson & 50,408 & 42 &, 175 \\
Desvianza & 34,806 & 42 &, 777 \\
\hline
\end{tabular}

Función de enlace: Logit.

Tabla 7: Pseudo R cuadrado de la prueba de regresión entre las dimensiones de los factores asociados al riesgo sobre la deserción estudiantil del Instituto Superior Tecnológico Público

\begin{tabular}{lc}
\hline Cox y Snell &, 139 \\
Nagelkerke &, 181 \\
McFadden &, 102 \\
\hline Función de enlace: Logit.
\end{tabular}

yente, no deja de ser importante señalar que para el caso de los factores socioeconómicos el nivel de riesgo medio estuvo cerca de ser significativo $(p=0,073)$. Este resultado se refrenda perfectamente con los coeficientes de Wald, los mismos que, al no tener significancia el p valor, entonces indicarían que ninguno de los factores de riesgo analizados puede predecir con un buen nivel de potencia a la variable de- 


\begin{tabular}{|c|c|c|c|c|c|c|c|c|}
\hline & \multicolumn{5}{|c|}{ Desv. } & \multicolumn{2}{|c|}{ Intervalo de confianza al $95 \%$} \\
\hline & & Estimación & Error & Wald & $\mathrm{gl}$ & Sig. & Límite inferior & Límite superior \\
\hline \multirow[t]{5}{*}{ Umbral } & $\begin{array}{l}{[\text { D_estud }=1} \\
\text { (Baja) }]\end{array}$ & $\begin{array}{c}-3,581 \\
\end{array}$ & 1,797 & 3,972 & 1 & 046 & $-7,102$ &,- 059 \\
\hline & $\begin{array}{l}{[\text { D_estud }=2} \\
(\text { Media })]\end{array}$ & 1,307 & 1,744 &, 562 & 1 &, 454 & $-2,112$ & 4,726 \\
\hline & $\begin{array}{l}{[\text { Personales }=1} \\
(\text { Baja })]\end{array}$ &,- 579 & 1,385 &, 175 & 1 &, 676 & $-3,295$ & 2,136 \\
\hline & $\begin{array}{l}{[\text { Personales }=2} \\
(\text { Media })]\end{array}$ &, 385 & 1,114 &, 119 & 1 &, 730 & $-1,798$ & 2,569 \\
\hline & $\begin{array}{l}{[\text { Personales }=3} \\
\text { (Alta) }]\end{array}$ & $0^{a}$ & . & . & 0 & . & . & . \\
\hline \multirow{9}{*}{ Ubicación } & (Academicos $=1$ & 1,282 & 1,282 & 1,000 & 1 &, 317 & $-1,231$ & 3,794 \\
\hline & $\begin{array}{l}{[\text { Academicos }=2} \\
(\text { Media })]\end{array}$ & 1,490 & 1,228 & 1,473 & 1 &, 225 &,- 916 & 3,897 \\
\hline & $\begin{array}{l}{[\text { Academicos }=3} \\
(\text { Alta })]\end{array}$ & $0^{a}$ & . & . & 0 & . & . & . \\
\hline & $\begin{array}{l}{[\text { Institucionales }=1} \\
(\text { Baja })]\end{array}$ & $-2,282$ & 1,562 & 2,133 & 1 & ,144 & $-5,344$ &, 780 \\
\hline & $\begin{array}{l}{[\text { Institucionales }=2} \\
(\text { Media })]\end{array}$ &,- 255 & ,722 &, 125 & 1 &, 724 & $-1,669$ & 1,160 \\
\hline & $\begin{array}{l}{[\text { Institucionales }=3} \\
(\text { Alta })]\end{array}$ & & . & . & 0 & . & . & . \\
\hline & $\begin{array}{l}{[\text { Socioeconomics }=} \\
(\text { Baja })]\end{array}$ & $1-1,328$ &, 844 & 2,477 & 1 & ,116 & $-2,982$ &, 326 \\
\hline & $\begin{array}{l}{[\text { Socioeconomics }=} \\
(\text { Media })]\end{array}$ & $2-1,289$ &, 718 & 3,223 & 1 & ,073 & $-2,696$ & ,118 \\
\hline & $\begin{array}{l}{[\text { Socioeconomics }=} \\
\text { (Alta) }]\end{array}$ & & . & . & 0 & . & . & . \\
\hline
\end{tabular}

Función de enlace: Logit

${ }^{a}$ Este parámetro está establecido en cero porque es redundante. 
serción. Puede ser también que el tamaño de muestra quizá haya determinado tener una no-significancia estadística. Esto será motivo de discusión más adelante.

\section{Discusión}

Según los resultados obtenidos de la variable factores de riesgo, los niveles de la variable fueron los siguientes: en el nivel riesgo medio se alcanzó un $92 \%$, en el nivel riesgo bajo se obtuvo un $5,3 \%$, y en el nivel alto un $2.7 \%$. Dichos resultados fueron distintos a los hallazgos alcanzados por Albán y Mauricio (2018) en su investigación sobre factores que influyen en la deserción universitaria en Ecuador, en el que se concluyó que las probabilidades de deserción fueron altas (57\%). La deserción es evidente, aunque en el caso de los institutos de educación superior tecnológicos públicos hay un predominio del factor de riesgo medio, mientras que en el caso de las instituciones de educación superior tecnológicos privados (Albán y Mauricio, 2018) tiene predominio el factor de deserción alto. Esto es así porque los estudiantes de los institutos privados, generalmente, son jóvenes que no ingresaron a las universidades por lo cual presentan una cierta desmotivación para seguir una carrera profesional. Asimismo, suelen ser jóvenes con dificultades académicas que han terminado la escuela secundaria y no han tenido la oportunidad de tener una preparación pre universitaria.

Por otra parte, se demostró que los factores de riesgo socioeconómicos no influyeron en la deserción estudiantil del Instituto de Educación Superior. A su vez, alcanzó un porcentaje considerable $(61.3 \%)$, similar a lo obtenido por Gravini (2016) en su investigación sobre la constancia, el abandono y la deserción en relación a un conjunto de pensamientos, la resiliencia y el beneficio académico de alumnos con problemas socioeconómicos. Este autor concluyó que una amenaza en la economía del estudiante aumenta la posibilidad de convertirse en desertor. Respecto a la dimensión factores de riesgo personales, se observó que ninguno de los niveles fue significativo. A su vez, el nivel de mayor frecuencia ( $76 \%$ ) fue el nivel medio. Este resultado concuerda con el obtenido por Ruiz (2018) en su investigación sobre abandono de estudiantes del primer ciclo en el Instituto de Educación Superior de Administración Señor de Sipán, quien finalizó poniendo en evidencia que los aspectos personales y un tiempo insuficiente para los estudios llevan hacia la deserción. En la dimensión factores de riesgo institucionales, se apreció que ninguno de los niveles fue significativo. A su vez, el nivel de mayor porcentaje con un $78.7 \%$ fue el nivel medio. Para Ruiz (2018), este resultado es relevante, ya que el problema se presenta en los primeros ciclos de estudios y el mayor motivo de deserción fueron directivas definidas en el 2014, pues para ser promovido un estudiante debe tener una cierta cantidad de asignaturas aprobadas. Esta directiva se consolidó en el año 2016 y se incrementó la deserción en este año.

Respecto a la variable deserción, se distinguió que la mayor frecuencia estuvo en el nivel deserción media, con un $69.3 \%$, con una tendencia definida hacia una alta deserción con un $26.7 \%$. Por otra parte, la deserción baja solamente tuvo un $4 \%$. 
Dichos resultados son congruentes con los hallados por Casanova, Cervero, Núñez, Almeida, y Bernardo (2018) en su estudio sobre factores que determinan la persistencia y deserción de los estudiantes universitarios, en España. De acuerdo con esto, el rendimiento académico es concluyente en la toma de decisión de continuar o abandonar los estudios. La decisión de permanecer o abandonar en la institución es altamente predictiva, con un mayor porcentaje de éxito en el caso de permanencia $(95.8 \%)$ que en el caso de deserción (65.6\%). A su vez, Alban y Mauricio (2018) en su investigación titulada Factores que influyen en la deserción universitaria, en Ecuador, concluyen definitivamente, que las probabilidades de deserción son elevadas $(57 \%)$, puesto que los jóvenes se dejan llevar por el entusiasmo de la educación superior, pero no tienen bien definida la carrera que deben estudiar. Entonces, cuando ya están estudiando en los primeros ciclos encuentran una realidad diferente a la que ellos esperaban, y ahí nace la desmotivación que los lleva a convertirse en estudiantes que desertan.

En la dimensión que está en relación con la institución se apreció que el mayor porcentaje se presentó en el nivel de deserción media (56\%). Asimismo, en la dimensión retiro temporal o definitivo de la institución la mayor frecuencia estuvo también en el nivel medio (69\%). En la dimensión de descontento con la institución se encontró que la mayor proporción se ubicó en el nivel alto con un $50.7 \%$, siendo la dimensión con mayor frecuencia alcanzada en este nivel de deserción. El estudiante muchas veces no se identifica con la institución, por que las instalaciones de los ambientes de estudio muchas veces no son las adecuados, así como los talleres y laboratorios muchas veces no son ambientes adecuados para el desarrollo de la carrera profesional que hayan elegido.

\section{Conclusiones}

En primer lugar, se concluye que en la variable deserción baja hubo significación $(p=0,005<0,05)$ y, a su vez, en contraste con el nivel deserción media, no presenta significancia. En la variable de factores de riesgo, se concluye que ninguno de los tres niveles fue significativo, por lo cual se afirma que no influyen en la deserción de estudiantes del primer y segundo ciclo del Instituto Superior Tecnológico Público.

En segundo lugar, se concluye respecto a la dimensión factores de riesgo personales que ninguno de los niveles fue significativo -ya que se mostró en el nivel bajo $(p=0,676>0,05)$ y en el nivel medio $(p=0,730>0,05)-$, por lo que se acepta la hipótesis nula que refiere que los factores de riesgo personales no influyen en la deserción de estudiantes del primer y segundo ciclo del Instituto de Educación Superior Tecnológico Público.

En tercer lugar, se concluye respecto a la dimensión factores institucionales que ninguno de los niveles fue significativo, en el nivel bajo $(p=0,144>0,05)$ y en el nivel medio $(p=0,724>0,05)$, por lo se acepta la hipótesis nula que refiere que los factores de riesgo institucionales no influyen en la deserción de estudiantes del primer y segundo ciclo del Instituto de Educación Superior Tecnológico Público.

En cuarto lugar, se concluye respecto a 
la dimensión factores académicos que ninguno de los niveles fue significativo, en el nivel bajo $(p=0,317>0,05)$ y en el nivel medio $(p=0,225>0,05)$ que, al ser mayor a la significancia esperada, se acepta la hipótesis nula que refiere que los factores de riesgo académicos no influyen en la deserción de estudiantes del primer y segundo ciclo del Instituto de Educación Superior Tecnológico Público.

Finalmente, se concluye respecto a la dimensión de factores socioeconómicos que ninguno de los niveles fue significativo, ya que en el nivel bajo $(p=0,116>$ $0,05)$ y en el nivel medio $(p=0,73>0,05)$ que, al ser mayor a la significancia esperada, permite aceptar la hipótesis nula que refiere que los factores de riesgo socioeconómicos no influyen en la deserción de estudiantes del primer y segundo ciclo del Instituto de Educación Superior Tecnológico Público.

\section{Referencias}

(1) Albán, M. y Mauricio D. (2018). Factors that influence undergraduate university desertion according to student's perspective. University of Cotopaxi.

(2) Bernal, C. (2006). Metodología de la Investigación. Pearson educación.

(3) Casanova, J. Cervero, A. Núñez, J. Almeida, L. y Bernardo, A. (2018). Factors that determine the persistence and dropout of university students. University of Minho and 2 University of Oviedo. Revista Psicothema, (304), Recu- perado de http://www.psicothema. $\mathrm{com} /$ psicothema.asp?id $=4501$

(4) Centro Interuniversitario de desarrollo (CINDA). (2006). Estudio sobre repitencia y deserción universitaria en América Latina 2006-05. Recuperado de https: //cinda.cl/wp-content/uploads/ 2006/05/repitencia-y-desercionuniversitaria-en-latinoamerica.pdf

(5) Corzo, D. D. (2018). Gestión administrativa y deserción de estudiantes en el instituto Julio Cesar Tello del distrito de Huarochiri-Lima, 2018. Tesis de maestría inédita. Universidad Cesar Vallejo.

(6) Econlink (2017). El Método Hipotético Deductivo. Recuperado el 12 de junio de 2019 de https: //www.econlink.com.ar/ciencia/ metodo-hipotetico-deductivo

(7) Fonseca, G., y García, F. (2016). Permanencia y abandono de estudios en estudiantes universitarios: un análisis desde la teoría organizacional. Revista de Educación Superior. 45(179). Recuperado de https://doi.org/10.1016/j. resu.2016.06.004

(8) Gravini, M. L. (2016). La permanencia y la deserción estudiantil y su relación con el autoconcepto, la resiliencia y el rendimiento académico en estudiantes con riesgo socioeconómico alto o bajo. Tesis doctoral. Universidad Autónoma de Madrid. 
(9) Instituto nacional de estadística e informática (2017). Censos nacionales 2017, Perfil sociodemográfico del Perú. Recuperado el 14 de junio de 2019, de https://cutt.ly/ MGukK0.

(10) Jiu, K. (2016). Factores de riesgo que desencadenan caries y enfermedad periodontal en pacientes adultos del consultorio médico El Buen Samaritano, Quebrada Retamal, Manchay, 2015. Tesis de maestría inédita. Universidad Cesar Vallejo.

(11) López, F. (2018). Educación superior comparada: Tendencias mundiales y de América Latina y el Caribe. Revista Sorocaba. 21(1) pp. 2021. Recuperado de https://cutt.ly/ pGyMYn

(12) McLeod, S. (2019). Qualitative vs. quantitative research. Recuperado de https://www.simplypsychology. org/qualitative-quantitative.html

(13) Merlos, G. (2017). School dropout for the adult learners into the Training Center for Industrial Work in Mexico. Inter-American Journal of Adult Education. 38(1). Recuperado de https://www.researchgate. net/publication/Work_in_Mexico

(14) Ministerio de educación (2014). Deserción escolar. Recuperado de http://escale.minedu.gob. pe/documents/10156/5232292/ Edudatos $+24+$ agosto +2016 .pdf

(15) Pérez, J. y Gardey, A. (2017). Definición de factores de riesgo. Gestión de wordpress. 1(1), Recuperado de https://definiciones.de/factoresde-riesgo/.

(16) Rafeedalli, E. (2014). Influence of self-concept and emotional maturity on leadership behaviour of heads of secondary schools in Kerala. Tesis doctoral. Kannur University.

(17) Rojas, M. (2008). El abandono de los estudios: deserción y decepción de la juventud. Revista Hologramática. (104). Recuperado de http: //cienciared.com.ar/ra/usr/3/467/ hologramatican10vol4pp7594.pdf

(18) Ruiz, M. A. (2018). Factores que influyen en la deserción de los alumnos del primer ciclo de educación a distancia en la Escuela de Administración de la Universidad Señor de Sipán. Períodos académicos 2011-1 al 2013-1: lineamientos para disminuir la deserción. Educación. 27(52), pp.160-173. Recuperado de https://doi.org/10.18800/ educacion.201801.009.

(19) Sagenmüller, I. (2019). Student retention: 8 reasons people drop out of higher education. U- planner. Recuperado el 5 de Julio de 2019, de https://www.u-planner.com/enus/home.

(20) Sánchez, H. y Reyes, M. (2002). Metodología y diseños en la investigación científica. Editorial Mantaro.

(21) Sistema Nacional de Evaluación y Acreditación de la Calidad en Educación (SINEACE), (2018). 
Solo $12 \%$ de jóvenes entre los 25 y 34 años culminan sus estudios técnicos. Recuperado de https: //www.sineace.gob.pe/solo-12-dejovenes-entre-los-25-y-34-anosculminan-sus-estudios-tecnicos/.

(22) Torres, L. (2016). Patrones de deserción estudiantil asociados a los datos operativos de los estudiantes en el instituto superior tecnológico Daniel Villar de Caraz-2016. Tesis de doctorado. Universidad Cesar Vallejo.

(23) Trevellini, B. (2018). Análisis de los factores de riesgo en la desvinculación en una nueva carrera en Administración y Contabilidad en una sede de la Universidad de la Repúbli$c a$. Tesis de maestría inédita. Universidad ORT.

(24) Valderrama, S. (2013). Pasos para elaborar proyectos y tesis de investigación científica. Editorial San Marcos.

(25) Viale, H. (2014). Una aproximación teórica a la deserción estudiantil universitaria. $R e$ vista RIDU. (81). Recuperado de https://dialnet.unirioja.es/ descarga/articulo/4898826.pdf.

(26) Wu L. (2018). Research designs: Non-Experimental vs Experimental. Recuperado el 21 de junio de 2019, de https://www.statisticssolutions. com/research-designs-nonexperimental-vs-experimental/. 